POS PROCEEDINGS

\title{
Studies of conventional quarkonia at Belle
}

\author{
Anatoly Sokolov*t \\ Institute for High Energy Physics, Protvino, Russia \\ E-mail: Sokolov_a@ihep.ru
}

Studies of conventional quarkonia using $250-400 \mathrm{fb}^{-1}$ of data collected with the Belle detector at the KEKB asymmetric-energy $e^{+} e^{-}$collider are presented.

International Europhysics Conference on High Energy Physics

July 21st - 27th 2005

Lisboa, Portugal

${ }^{*}$ Speaker.

${ }^{\dagger}$ On behalf of the Belle Collaboration 


\section{Study of charmonia in four-meson final states produced in two-photon collisions}

We have measured the production of the charmonium states, $\eta_{c}(1 S), \chi_{c 0}$ and $\chi_{c 2}$, from twophoton collisions in decay modes to four-meson final states, $\pi^{+} \pi^{-} \pi^{+} \pi^{-}, K^{+} K^{-} \pi^{+} \pi^{-}$and $K^{+} K^{-} K^{+} K^{-}$, with the Belle detector. We have also searched for production of $\eta_{c}(2 S)$ in the same final states. All the results of this study are preliminary.

We used $280 \mathrm{fb}^{-1}$ of data recorded by the Belle detector [1] at the KEKB asymmetric-energy $e^{+} e^{-}$collider [2]. The candidate events are selected by requiring four charged particles with the zero net charge. The analysis is performed in the "no-tag" mode, where neither the recoil electron nor positron is detected. The vector sum of four tracks transverse momenta with respect to the $e^{+} e^{-}$ -beam axis in the c.m. frame, $\Sigma \mathbf{p}_{t}^{*}$, is less $0.1 \mathrm{GeV} / c . K / \pi$ separation is made using a likelihood ratio from the $\mathrm{ACC}$, TOF and $\mathrm{CDC}$ information [1].

In the following analysis, we take only the combinations in which the net strangeness is conserved, $\pi^{+} \pi^{-} \pi^{+} \pi^{-}, K^{+} K^{-} \pi^{+} \pi^{-}$and $K^{+} K^{-} K^{+} K^{-}$. We determine the yields of charmonium events using the invariant mass distributions in each of the three final states. We find clear enhancements at the $\eta_{c}(1 S)$ at $\sim 2.98 \mathrm{GeV} / c^{2}, \chi_{c 0}$ at $\sim 3.41 \mathrm{GeV} / c^{2}$, and $\chi_{c 2}$ at $\sim 3.555 \mathrm{GeV} / c^{2}$ in all the final states. We do not see any clear signatures of $\eta_{c}(2 S)$. We study quasi-two-body decays of charmonia, in which a charmonium meson decays into two resonances, and each resonance decays to two final-state mesons.

The present and previous measurements $[3,4]$ give the "direct" $\mathscr{G} \equiv \Gamma_{\gamma \gamma} \mathscr{B}$ parameters for the processes discussed here. "Indirect" comparisons are also possible by converting the $\mathscr{G}$ parameter measured for a certain decay mode to the present measured mode using the ratio of the branching fractions, $\mathscr{G}(R \rightarrow X)=\mathscr{G}(R \rightarrow A) \cdot(\mathscr{B}(R \rightarrow X) / \mathscr{B}(R \rightarrow A))$, where $R \rightarrow A$ is a "normalization" process for which previous measurement(s) are available. The results for $\mathscr{G}$ are summarized in Table 1. In the $\eta_{c}$ results, we find large systematic deviations between the present and previous results. In contrast, all the $\chi_{c 0}$ and $\chi_{c 2}$ results show good agreement. No clear signature for the $\eta_{c}(2 S)$ is seen in these decay processes. We find prominent two-body $\eta_{c}(1 S)$ and $\chi_{c 2}$ decays to $K^{*}(892)^{0} \bar{K}^{*}(892)^{0}$ as well as $\eta_{c}(1 S), \chi_{c 0}$ and $\chi_{c 2}$ decays to $\phi \phi$. In addition, we observe $\eta_{c}(1 S) \rightarrow$ $f_{2}(1270) f_{2}(1270)$.

\section{Study of Charmonium Decays into Baryon-Antibaryon Pairs}

Visible structures in the mass spectra of a baryon-antibaryon pair of three-body $B$ decays arise from charmonium decays. There is particular interest in $J / \psi \rightarrow p \bar{p}$, where the proton angular distribution has been studied. The baryon angular distribution can be parameterized as $\sim 1+$ $\alpha \cos ^{2} \theta$, where $\theta$ is the baryon polar angle in the $J / \psi$ helicity frame.

In this study of two-body baryonic decays of charmonia we focus on the decay processes, $B^{+} \rightarrow p \bar{p} K^{+}$and $B^{+} \rightarrow \Lambda \bar{\Lambda} K^{+}$. We use a $350 \mathrm{fb}^{-1}$ data sample, consisting of $386 \times 10^{6} B \bar{B}$ pairs, collected by the Belle detector [1].

To isolate the signal and suppress the dominant background arising from the continuum $e^{+} e^{-} \rightarrow$ $q \bar{q}$ process we follow the scheme suggested in Ref. [6]. An unbinned likelihood fit is used to estimate the $B$ yield. There are clear $\eta_{c}$ and $J / \psi$ peaks in the $M_{p}{ }^{-}$mass spectrum. The measured branching fractions for charmonia decaying into $p \bar{p}$ are $\mathscr{B}\left(\eta_{c} \rightarrow p \bar{p}\right)=(1.58 \pm 0.12($ stat $) \pm$ 
Table 1: The present results of the $\mathscr{G} \equiv \Gamma_{\gamma \gamma} \mathscr{B}$ and comparisons with previous measurements (shown as "direct" and "indirect", see text) [5].

\begin{tabular}{|c|c||c|c|}
\hline Process & $\begin{array}{c}\text { present } \\
\mathscr{G}(\mathrm{eV})\end{array}$ & $\begin{array}{c}\text { direct } \\
\mathscr{G}(\mathrm{eV})\end{array}$ & $\begin{array}{c}\text { indirect } \\
\mathscr{G}(\mathrm{eV})\end{array}$ \\
\hline$\eta_{c} \rightarrow \pi^{+} \pi^{-} \pi^{+} \pi^{-}$ & $26.4 \pm 3.7 \pm 3.2$ & $180 \pm 70 \pm 20$ & $101 \pm 40$ \\
$\eta_{c} \rightarrow K^{+} K^{-} \pi^{+} \pi^{-}$ & $23.7 \pm 3.4 \pm 2.8$ & $210 \pm 70$ & $126 \pm 64$ \\
$\eta_{c} \rightarrow K^{+} K^{-} K^{+} K^{-}$ & $7.3 \pm 1.6 \pm 1.0$ & $280 \pm 70$ & $13 \pm 7$ \\
$\eta_{c} \rightarrow f_{2} f_{2}$ & $72 \pm 24 \pm 12$ & - & - \\
$\eta_{c} \rightarrow K^{*} \bar{K}^{*}$ & $35 \pm 6 \pm 5$ & - & $72 \pm 34$ \\
$\eta_{c} \rightarrow f_{2} f_{2}^{\prime}$ & $34 \pm 12 \pm 6$ & - & - \\
$\eta_{c} \rightarrow \phi \phi$ & $7.0 \pm 1.4 \pm 1.3$ & - & $21 \pm 10$ \\
\hline$\chi_{c 0} \rightarrow \pi^{+} \pi^{-} \pi^{+} \pi^{-}$ & $48.8 \pm 5.9 \pm 4.4$ & $75 \pm 13 \pm 8$ & $67 \pm 14$ \\
$\chi_{c 0} \rightarrow K^{+} K^{-} \pi^{+} \pi^{-}$ & $39.9 \pm 4.8 \pm 4.0$ & - & $55 \pm 18$ \\
$\chi_{c 0} \rightarrow K^{+} K^{-} K^{+} K^{-}$ & $8.7 \pm 1.5 \pm 1.2$ & - & $6.0 \pm 2.0$ \\
$\chi_{c 0} \rightarrow K^{* 0} K^{-} \pi^{+}+c . c$. & $16.4 \pm 6.1 \pm 2.5$ & - & $31 \pm 13$ \\
$\chi_{c 0} \rightarrow \phi \phi$ & $2.5 \pm 1.0 \pm 0.4$ & - & $2.6 \pm 1.7$ \\
\hline$\chi_{c 2} \rightarrow \pi^{+} \pi^{-} \pi^{+} \pi^{-}$ & $4.73 \pm 0.49 \pm 0.57$ & $6.4 \pm 1.8$ & $7.7 \pm 1.5$ \\
$\chi_{c 2} \rightarrow K^{+} K^{-} \pi^{+} \pi^{-}$ & $4.92 \pm 0.54 \pm 0.59$ & - & $6.4 \pm 1.9$ \\
$\chi_{c 2} \rightarrow K^{+} K^{-} K^{+} K^{-}$ & $1.23 \pm 0.20 \pm 0.17$ & - & $0.94 \pm 0.29$ \\
$\chi_{c 2} \rightarrow \rho^{0} \pi^{+} \pi^{-}$ & $3.9 \pm 1.9 \pm 0.5$ & - & $3.6 \pm 2.1$ \\
$\chi_{c 2} \rightarrow K^{*} \bar{K}^{*}$ & $2.61 \pm 0.54 \pm 0.89$ & - & - \\
$\chi_{c 2} \rightarrow \phi \phi$ & $0.63 \pm 0.22 \pm 0.17$ & - & \\
\hline
\end{tabular}

$0.22($ syst $) \pm 0.47(\mathrm{PDG})) \times 10^{-3}$ and $\mathscr{B}(\mathrm{J} / \psi \rightarrow p \bar{p})=(2.24 \pm 0.13($ stat $) \pm 0.31($ syst $) \pm 0.01(\mathrm{PDG})) \times$ $10^{-3}$. $\alpha$ is determined from the fit of the $\cos \theta$ distribution for $J / \psi$ candidates to be $0.54 \pm 0.14$. From the baryon-antibaryon mass spectrum in $B^{+} \rightarrow \Lambda \bar{\Lambda} K^{+}$decays we estimate the branching fraction of $\eta_{c} \rightarrow \Lambda \bar{\Lambda}$ and $J / \psi \rightarrow \Lambda \bar{\Lambda}$. The results are $\mathscr{B}\left(\eta_{c} \rightarrow \Lambda \bar{\Lambda}\right)=\left(0.87_{-0.21}^{+0.24}(\right.$ stat $) \pm 0.14($ syst $) \pm$ $0.27(\mathrm{PDG})) \times 10^{-3}$ and $\mathscr{B}(\mathrm{J} / \psi \rightarrow \Lambda \bar{\Lambda})=\left(2.00_{-0.29}^{+0.33}(\right.$ stat $) \pm 0.34($ syst $\left.) \pm 0.08(\mathrm{PDG})\right) \times 10^{-3}$.

\section{Search for the $h_{c}$ meson in $B^{+} \rightarrow h_{c} K^{+}$}

The $h_{c}$ meson is the $1^{1} P_{1}$ spin singlet state of $c \bar{c}$, which is expected to be a narrow resonance $\left(\Gamma_{h_{c}}<1.1 \mathrm{MeV} / c^{2}\right)$ that lies between $J / \psi(1 S)$ and $\psi(2 S)$. Recently, CLEO[7] has reported the observation of $h_{c} \rightarrow \gamma \eta_{c}$ at a mass of $M=3524.4 \pm 0.6 \pm 0.4 \mathrm{MeV} / c^{2}$.

M. Suzuki [8] has proposed using the decay chain $B \rightarrow h_{c} K, h_{c} \rightarrow \eta_{c} \gamma$ to look for the $h_{c}$ meson. Measurement of the branching fraction for $B \rightarrow h_{c} K$ will provide useful information on non-factorizable contributions in $B$ to charmonium decays.

Here we present the results of a search for $B^{+} \rightarrow h_{c} K^{+}, h_{c} \rightarrow \eta_{c} \gamma$ with $\eta_{c} \rightarrow K_{S}^{0} K^{ \pm} \pi^{\mp}$ and $p \bar{p}$ using a $253 \mathrm{fb}^{-1}$ data sample, which contains $275 \times 10^{6}$ produced $B \bar{B}$ pairs, collected with the Belle detector[1]. To isolate the signal and suppress the large background from continuum $e^{+} e^{-} \rightarrow q \bar{q}$ 
we follow the scheme suggested in Ref. [6]. No significant signals are seen for $3.17<M\left(\eta_{c} \gamma\right) \leq$ $3.67 \mathrm{GeV} / c^{2}$. To obtain upper limits on the branching fractions for $B^{+} \rightarrow \eta_{c} \gamma K^{+}$we combine the likelihoods for the $\eta_{c} \rightarrow K_{S}^{0} K^{-} \pi^{+}$and $\eta_{c} \rightarrow p \bar{p}$ modes. Assuming $\mathscr{B}\left(h_{c} \rightarrow \eta_{c} \gamma\right)=0.5$, we obtain $90 \%$ C.L. upper limits on branching fractions for $B^{+} \rightarrow h_{c} K^{+}$as a function of the $h_{c}$ mass. For $M_{h_{c}}=3.527 \mathrm{GeV} / c^{2}$, we find $\mathscr{B}\left(B^{+} \rightarrow h_{c} K^{+}\right)<3.8 \times 10^{-5}$. This is consistent, but only barely, with the lower bound on the $B \rightarrow h_{c} K$ branching fraction obtained by Colangelo, Fazio and Pham [9], which is $\mathscr{B}\left(B \rightarrow h_{c} K\right)=(2-12) \times 10^{-4}$.

\section{Search for the decay $\Upsilon(4 S) \rightarrow \Upsilon(1 S) \pi^{+} \pi^{-}$}

The bottomonium state $\Upsilon(4 S)$ has a mass above the threshold for $B \bar{B}$ pair production and decays mainly into these $B$-meson pairs $(\mathscr{B}(\Upsilon(4 S) \rightarrow B \bar{B})>96 \%$ [5]). Here we report the first evidence for the non- $B \bar{B}$ decay mode $\Upsilon(4 S) \rightarrow \Upsilon(1 S) \pi^{+} \pi^{-}$from the Belle experiment.

In this study $398 \mathrm{fb}^{-1}$ of data collected by the Belle detector [1] on the $\Upsilon(4 S)$ resonance and in the nearby continuum is used. Well reconstructed charged particles are used to reconstruct the decay $\Upsilon(4 S) \rightarrow \Upsilon(1 S) \pi^{+} \pi^{-}$with the subsequent leptonic decay $\Upsilon(1 S) \rightarrow \ell^{+} \ell^{-}$.

$\mu^{+} \mu^{-} \pi^{+} \pi^{-} X$ events with an invariant mass $M_{\mu^{+} \mu^{-}}>9 \mathrm{GeV} / c^{2}$ were selected from the sample selected by the standard Belle hadronic selection. The muon pair invariant mass distribution, $M_{\mu^{+} \mu^{-}}$, for the $\mu^{+} \mu^{-} \pi^{+} \pi^{-} X$ events has a clear low background signal for $\Upsilon(1 S) \rightarrow \mu^{+} \mu^{-}$. To observe resonance states that decay into the $\Upsilon(1 S) \pi^{+} \pi^{-}$final state the distribution of the mass difference $\Delta M=\left(M_{\mu^{+} \mu^{-} \pi^{+} \pi^{-}}-M_{\mu^{+} \mu^{-}}\right)$where $M_{\mu^{+} \mu^{-}}$is restricted to $\left|M_{\mu^{+} \mu^{-}}-M_{\Upsilon(1 S)}\right|<6 \mathrm{MeV} / c^{2}$ was examined. Three peaks exist in the $\Delta M$ distribution. The first (second, third) peaks correspond to $\Delta M \sim 0.56(0.89,1.12) \mathrm{GeV} / c^{2}$, respectively. The first and second peaks originate from the decays $\Upsilon(2 S, 3 S) \rightarrow \Upsilon(1 S) \pi^{+} \pi^{-}$with a subsequent $\Upsilon(1 S) \rightarrow \mu^{+} \mu^{-}$transition, respectively. The position of the third peak, $\Delta M=(1119.0 \pm 1.4) \mathrm{MeV} / c^{2}$, is in good agreement with the mass difference $\left(M_{\Upsilon(4 S)}-M_{\Upsilon(1 S)}\right)$ from the PDG [5]. The signal above background is determined from the fit to be, $N_{e v}=(38 \pm 6.9)$, with a statistical significance of 7.3 standard deviations. This peak is interpreted as a signal from the decay $\Upsilon(4 S) \rightarrow \Upsilon(1 S) \pi^{+} \pi^{-}$with a subsequent $\Upsilon(1 S) \rightarrow \mu^{+} \mu^{-}$ transition. This is the first example of a non- $B \bar{B}$ decay of the $\Upsilon(4 S)$ resonance. The preliminary result for the branching fraction is $\mathscr{B}\left(\Upsilon(4 S) \rightarrow \Upsilon(1 S) \pi^{+} \pi^{-}\right)=(1.1 \pm 0.2$ (stat.) \pm 0.4 (syst.) $) \cdot 10^{-4}$.

\section{References}

[1] Belle Collaboration, A. Abashian et al., Nucl. Inst. and Meth. A 479 (2002) 117.

[2] S. Kurokawa and E. Kikutani, Nucl. Inst. and Meth. A 499 (2003) 1.

[3] DELPHI Collaboration, J. Abdallah et al., Eur. Phys. Journ. C 31 (2003) 481.

[4] CLEO Collaboration, B.I. Eisenstein et al., Phys. Rev. Lett. 87 (2001) 061801.

[5] S. Eidelman et al., Particle Data Group, Phys. Lett. B 592 (2004) 1.

[6] Belle Collaboration, K. Abe et al., Phys. Lett. B 517 (2001) 309.

[7] CLEO Collaboration, J.L. Rosner et al., Phys. Rev. Lett. 95 (2005) 102003.

[8] M. Suzuki, Phys. Rev. D 66 (2002) 037503.

[9] P. Colangelo, F. De Fazio and T.N. Pham, Phys. Rev. D 69 (2004) 054023. 